\title{
Discrete Geodesic Parallel Coordinates
}

\author{
HUI WANG, DLUT, TU Wien \\ DAVIDE PELLIS, TU Wien \\ FLORIAN RIST, KAUST, TU Wien \\ HELMUT POTTMANN, KAUST, TU Wien \\ CHRISTIAN MÜLLER, TU Wien
}

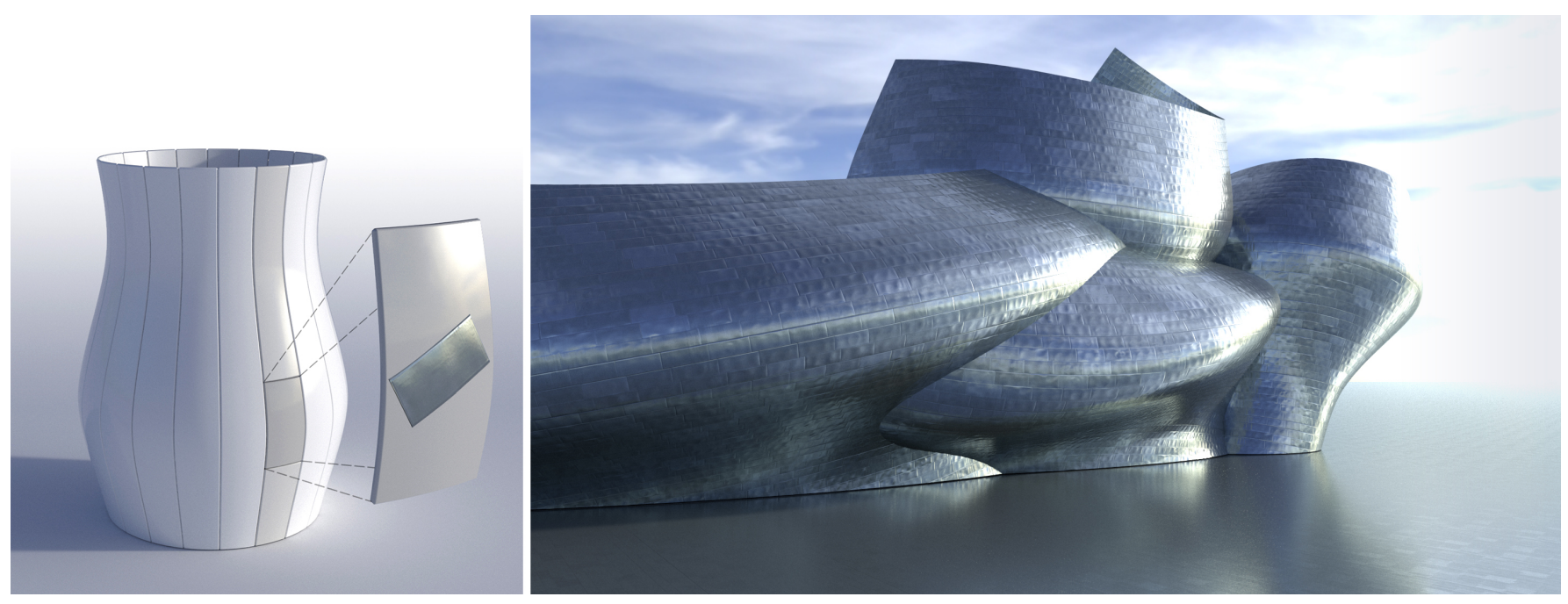

Fig. 1. Architectural design in reference to designs by architect Frank Gehry. In our case the four surface patches are not developable, but are isometric to the same surface of revolution. The rectangular metal sheets covering the surface can be produced using just one comparably small sector between two meridian curves of the corresponding surface of revolution as a mold (left and Figure 16). The long vertical strip (left; gray) of the surface of revolution is either one big mold or can be segmented into smaller molds if necessary. Metal sheets covering surface areas with almost vanishing Gaussian curvature can be produced without a special mold, i.e., as developable surface patches.

Geodesic parallel coordinates are orthogonal nets on surfaces where one of the two families of parameter lines are geodesic curves. We describe a discrete version of these special surface parameterizations and show that they are very useful for specific applications, most of which are related to the design and fabrication of surfaces in architecture. With the new discrete surface model, it is easy to control strip widths between neighboring geodesics. This facilitates tasks such as cladding a surface with strips of originally straight flat material or designing geodesic gridshells and timber rib shells. It is also possible to model nearly developable surfaces. These are characterized by geodesic strips with almost constant strip widths and are used for generating shapes that can be manufactured from materials which allow for some stretching or shrinking like felt, leather, or thin wooden

Authors' addresses: Hui Wang, DLUT, TU Wien; Davide Pellis, TU Wien; Florian Rist, KAUST, TU Wien; Helmut Pottmann, KAUST, TU Wien; Christian Müller, TU Wien.

Permission to make digital or hard copies of all or part of this work for personal or classroom use is granted without fee provided that copies are not made or distributed for profit or commercial advantage and that copies bear this notice and the full citation on the first page. Copyrights for components of this work owned by others than ACM must be honored. Abstracting with credit is permitted. To copy otherwise, or republish, to post on servers or to redistribute to lists, requires prior specific permission and/or a fee. Request permissions from permissions@acm.org.

(c) 2019 Association for Computing Machinery.

0730-0301/2019/9-ART \$15.00

https://doi.org/10.1145/nnnnnnn.nnnnnnn boards. Most importantly, we show how to constrain the strip width parameters to model a class of intrinsically symmetric surfaces. These surfaces are isometric to surfaces of revolution and can be covered with doubly-curved panels that are produced with only a few molds when working with flexible materials like metal sheets.

CCS Concepts: • Computing methodologies $\rightarrow$ Shape modeling; Optimization algorithms.

Additional Key Words and Phrases: discrete differential geometry, architectural geometry, computational fabrication, paneling, geodesic, geodesic strip, isometry, geodesic parallel coordinates

\section{ACM Reference Format:}

Hui Wang, Davide Pellis, Florian Rist, Helmut Pottmann, and Christian Müller. 2019. Discrete Geodesic Parallel Coordinates. ACM Trans. Graph. 1, 1 (September 2019), 13 pages. https://doi.org/10.1145/nnnnnnn.nnnnnnn

\section{INTRODUCTION}

Recent research in computational design incorporates key aspects of function and fabrication into an intelligent shape modeling process. This reduces time and cost of product development, but also offers totally new ways of combining digital design and fabrication. One such direction of research is material-aware shape modeling, and a natural assumption to start with is a flat sheet of material. If this 
material is much more easily bent than stretched, the ideal modelled shapes are developable surfaces. Interactive modeling with these surfaces is still an active topic of research (see, e.g., [Rabinovich et al. 2018a,b; Stein et al. 2018]). Recent work beyond developable surfaces gains design flexibility with a special class of auxetic materials [Konaković et al. 2016, 2018]. However, one does not directly obtain a watertight surface skin. A remarkable way of building watertight 3D surfaces from flat sheets is the use of planar rod networks embedded in pre-stretched fabric that deploy into complex, three-dimensional shapes [Perez et al. 2017]. The introduction of folds, straight or curved, is yet another way for building spatial structures from flat material. We do not review the wealth of research in this direction in detail and just refer to [Callens and Zadpoor 2018; Demain and O’Rourke 2007; Dudte et al. 2016].

Our work also deals with surfaces from flat sheets of material. It goes beyond developable surfaces and achieves watertight surfaces through an appropriate assembly of patches or panels. Motivated by applications in architecture, we pursue several closely connected goals: (i) We want to cover surfaces with originally nearly straight flat strips of material. (ii) By keeping the width of these strips almost constant, we want to get access to nearly developable surfaces. (iii) We aim at modelling surfaces with intrinsic symmetries. As intrinsic symmetries are not easily seen by a viewer, these surfaces still appear very much like freeform shapes. Our intrinsically symmetric design surfaces, such as the ones in Figures 1 and 2, are isometric to surfaces of revolution. We show that they offer new ways of fabricating surfaces, not just by bending flat sheets. One can cover them with doubly curved panels that are produced with only a small number of molds.

\subsection{Overview and contributions}

We introduce and study a new type of quad meshes which are discrete versions of surface parameterizations $f(u, v)$ with the attractive property that one family of iso-parameter curves $v=$ const are geodesics and the iso-parameter lines $u=$ const are orthogonal to them (Section 2). This extends recent research modeling discrete developable surfaces with the help of two orthogonal families of geodesics [Rabinovich et al. 2018a,b]. Whereas they model globally developable surfaces, we consider at first surfaces which allow for geodesic parallel coordinates, which are (locally) almost all surface patches. Our focus lies on modeling the families of geodesics. Through our ability to control the so called geodesic strip widths and by setting it to be constant we actually include the class of developable surfaces. Furthermore, whereas the focus of the work by Rabinovich et al. is the developable surface itself and not its parametrization, in our case the family of geodesic parameter lines is a significant part of the result.

The new discrete surfaces naturally decompose a surface into strips bounded by geodesics. The width of the strips is nicely controllable with help of the curves orthogonal to them and gives us a tool for modeling and representing nearly developable surfaces (Section 3). Our meshes provide a new effective scheme for designing geodesic strip layouts on a given reference surface. Even more importantly, we are now able to design the surface and the strip model simultaneously. This has the significant advantage that only

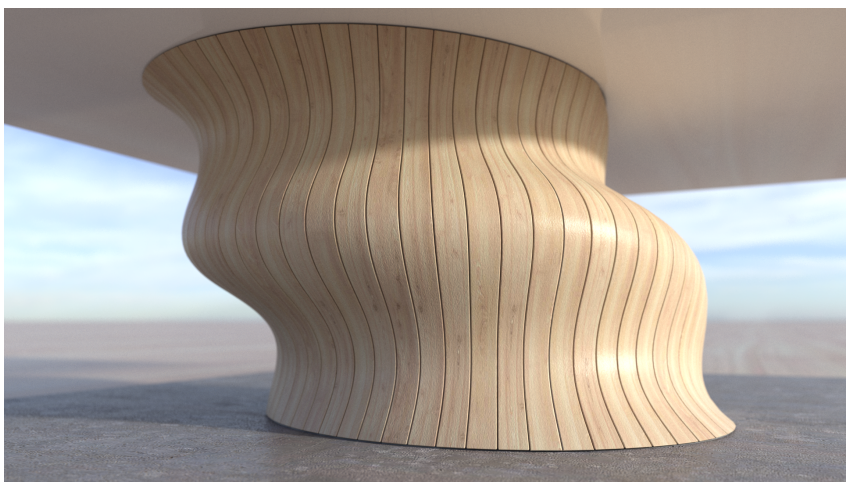

Fig. 2. Rendering of a wooden wall cladding. The underlying surface is isometric to a surface of revolution. It can be realized by attaching a sequence of bent timber strips. All strips develop to the same planar shape.

those surfaces are generated which fulfill given constraints on the layout of the strip model, for example, on the strip width variation.

A geodesic strip model can be realized joining together developable surface strips whose planar unfoldings are nearly straight. The strip width is in general not constant and thus different strips will have different unfoldings. However, there are surfaces for which all strips are congruent to each other in the unfolding. One such instance is provided by rotational strip models, obtained by cutting a rotational surface along regularly arranged planes through the rotational axis and connecting consecutive slices by developable (cylindrical) strips. Any isometrically deformed version of such a rotational strip model also enjoys the property of being composed of essentially only one type of a flat strip (see Fig. 2). In Section 4, we show how to model these intrinsically repetitive surfaces. While the recognition of a surface with this property could be performed with prior work on Killing vector fields [Ben-Chen et al. 2010], we are not aware of any contribution to the design of surfaces with this type of intrinsic symmetry.

In Section 5 we show how the new type of constrained meshes can be effectively computed via a numerical optimization scheme following [Tang et al. 2014] and provide some details on its implementation.

Various applications in architectural geometry such as geodesic gridshells, cladding and paneling are presented in Section 6. A remarkable consequence of intrinsic symmetry (isometry to a surface of revolution) is that it significantly extends the possibilities for paneling architectural skins with bendable material like sheet metal. We are not confined to developable panels anymore in order to obtain a cost effective solution, but can produce doubly-curved panels from just a few molds in the form of segments of the corresponding surface of revolution.

Finally, we discuss limitations and point to promising directions for future research.

\subsection{Related Work}

Most closely related to our research is recent work by Rabinovich et al. [2018a; 2018b]. It carefully studies a discrete model for developable surfaces, based on two orthogonal families of geodesics. The 
demonstrated advantages of this important special case of discrete geodesic parallel coordinates indicate the potential of our more general setting.

Geodesic parallel coordinates are closely tied to distance functions on surfaces. There is a huge amount of prior work on the related tasks of computing distance functions and geodesics on surfaces. We mention here only two of them, the fast marching method [Sethian 1999] and the highly efficient heat method [Crane et al. 2017]. Note that it is not our goal to provide another method for computing geodesics or distance fields. We want to model surfaces in a special representation which includes a family of geodesics and we want to control this family for various applications.

Part of our work concerns surfaces with a continuous intrinsic symmetry. Such surfaces can be characterized via non-trivial Killing fields. Discrete approximate Killing fields have been employed for various tasks in Geometry Processing, such as pattern design on surfaces [Ben-Chen et al. 2010] and discovery of intrinsic primitives [Solomon et al. 2011]. A large body of research deals with discrete intrinsic symmetries, e.g., [Ovsjanikov et al. 2008; Raviv et al. 2010].

The study of strip models for enhancing the understanding of differential geometric concepts and the associated construction of physical models is a classical topic of difference geometry [Sauer 1970], a precursor of modern discrete differential geometry. We point in particular to the pioneering work of S. Finsterwalder [1899], which explains remarkable relations between geometry and mechanics, and is of interest for various topics in computational design and fabrication. Much more recently, so-called semi-discrete surfaces received interest, both from the practical and theoretical perspective [Carl 2017; Müller and Wallner 2013; Pottmann et al. 2008] These surfaces are composed of strips of ruled surfaces, in particular developable surfaces, and constitute a type of surfaces that is situated between the purely discrete and the smooth setting. Most relevant for our work are the geodesic strip models. The design of such models of geodesic strips can follow a procedure for the layout of patterns formed by geodesics. It has applications in architecture and ship building [Kahlert et al. 2011; Pottmann et al. 2010]. Other methods like [Mitani and Suzuki 2004; Tang et al. 2016] provide methods to subdivide surfaces into regions are then represented by developable surface patches.

Our work is inspired by applications in architecture. The cladding of architectural skins with metal tiles is prominently featured in the work of Frank Gehry [Shelden 2002]. Cladding of a curved surface by bending nearly straight wooden panels has been applied at the wood ceiling in the Burj Khalifa office lobby in Dubai [Meredith and Kotronis 2012]. Appropriate manufacturing technologies for covering freeform surfaces with long curved metal panels have been developed by [Schneider and Mehrtens 2012]. Curved structures which arise from originally straight elements appear in various types of gridshells [Hernandez 2015], in particular geodesic gridshells and timber rib shells (see [Pirazzi and Weinand 2006] and various articles in [Bianconi and Filippucci 2019]).

A major problem in the realization of architectural freeform geometry is the diversity of constructional elements. Hence, various ways to achieve repetitive elements have been investigated (see the overview in [Pottmann et al. 2015; Schling 2018; Schling et al. 2018]). Even if different elements, e.g. panels, may not be congruent, they may be produced with the same mold or machine configuration, thus contributing to cost saving [Eigensatz et al. 2010]. To the best of our knowledge, intrinsic repetition by design is a new direction to simplify the construction of architectural freeform skins.

\section{DISCRETE GEODESIC PARALLEL COORDINATES}

In this section, we quickly recall some well known facts regarding geodesic parallel coordinates on surfaces and then present our discretization.

\subsection{Smooth geodesic parallel coordinates}

A family of parallel straight lines in the plane has the property that the distance between any two of them is constant. This property obviously does not characterize parallel straight lines as it also holds, e.g., for a family of concentric circles, or more generally for a family of offsets of a curve. The curves orthogonal to an offset family are straight lines and at least locally, these two curve families can be the iso-parameter lines of a parametrization $f(u, v)$ of a planar domain. This is a special case of geodesic parallel coordinates (Def. 2.1) on surfaces (see, e.g., [Kühnel 2003, Def. 4.27]). Recall that geodesic curves on surfaces are curves of (locally) shortest lengths.

Definition 2.1. A surface $f: \mathbb{R}^{2} \supseteq\left[u_{0}, u_{1}\right] \times\left[v_{0}, v_{1}\right] \rightarrow \mathbb{R}^{3}$ is parametrized along geodesic parallel coordinates if the parameter lines are orthogonal $\left(f_{u} \perp f_{v}\right)$ and one family of parameter lines, say the $u$-lines $(v=$ const), are geodesics, see Figure 3 (left).

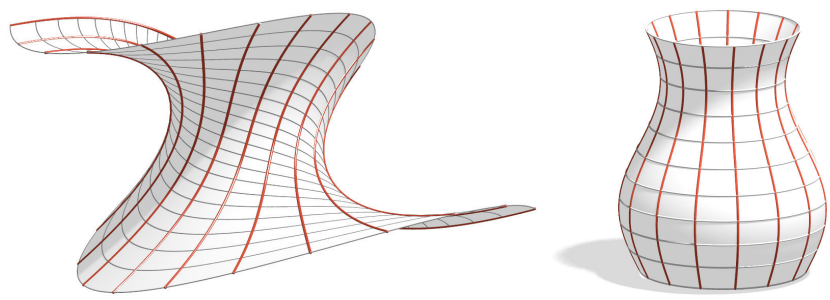

Fig. 3. Left: A surface parametrized by geodesic parallel coordinates. The red parameter lines are geodesics and the gray parameter lines are orthogonal to them. Right: A surface of revolution in its common parametrization is always parametrized by geodesic parallel coordinates. The meridian curves (red) are geodesics.

A very simple but nevertheless very important family of examples of such geodesic parallel coordinates consists of all surfaces of revolution given by the parametrization:

$$
f(u, v)=(r(u) \cos v, r(u) \sin v, h(u)) .
$$

This parametrization is clearly orthogonal and the meridian curves (Figure 3 (right) in red; $v=$ const) are geodesics.

\subsection{Discrete geodesic parallel coordinates}

In our discretization of geodesic parallel coordinates we generalize the approach of modeling developable nets with discrete orthogonal geodesic nets [Rabinovich et al. 2018a,b]. We will mainly be working with quadrilateral nets with regular combinatorics, i.e., like a rectangular portion of the $\mathbb{Z}^{2}$ lattice. 

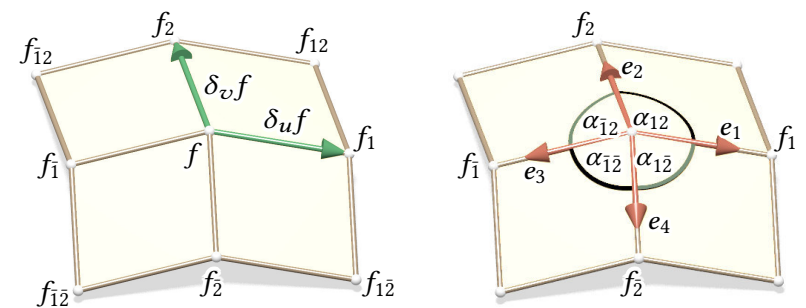

Fig. 4. Notation. Left: To make formulas shorter and clearer we leave away parameters $f(u, v) \rightarrow f$ and use the shift notation $f_{1}=f(u+1, v)$ and $f_{\overline{1}}=f(u-1, v)$. We denote edge vectors by $\delta_{u} f$ for $f_{1}-f$. Right: Additionally, we denote the unit length edge vectors emanating from $f$ by $e_{i}$ and angles between neighboring edges by $\alpha_{i j}$.

2.2.1 Notation. A discrete net is defined by a map $f: \mathbb{Z}^{2} \rightarrow \mathbb{R}^{3}$. The discrete parameter lines through $(u, v) \in \mathbb{Z}^{2}$ are polylines $f(\mathbb{Z}, v)$ and $f(u, \mathbb{Z})$ which we call $u$-lines and $v$-lines like in the smooth case.

Since we are mainly considering local properties of a net around $f(u, v)$, we use the following abbreviations for the vertices, similarly to [Rabinovich et al. 2018a]:

$$
\begin{aligned}
& f=f(u, v), \quad f_{1}=f(u+1, v), \quad f_{2}=f(u, v+1), \\
& f_{\overline{1}}=f(u-1, v), \quad f_{\overline{2}}=f(u, v-1), \quad f_{12}=f(u+1, v+1),
\end{aligned}
$$

etc. For an illustration of the notation see Figure 4 . The discrete partial derivatives are denoted with the first forward difference operator

$$
\delta_{u} f=f_{1}-f, \quad \delta_{u} f_{\overline{1}}=f-f_{\overline{1}}, \quad \delta_{v} f=f_{2}-f, \quad \text { etc, }
$$

and the second derivative accordingly

$$
\delta_{u u}\left(f_{\overline{1}}\right)=\delta_{u}\left(\delta_{u} f_{\overline{1}}\right)=\delta_{u}\left(f-f_{\overline{1}}\right)=\delta_{u} f-\delta_{u} f_{\overline{1}}=f_{1}-2 f+f_{\overline{1}} .
$$

Assuming non-degenerate edges (which is guaranteed through fairness terms in our algorithm), we additionally denote the unit edge vectors emanating from $f$ by

$$
e_{1}=\frac{\delta_{u} f}{\left\|\delta_{u} f\right\|}, \quad e_{2}=\frac{\delta_{v} f}{\left\|\delta_{v} f\right\|}, \quad e_{3}=-\frac{\delta_{u} f_{\overline{1}}}{\left\|\delta_{u} f_{\overline{1}}\right\|}, \quad e_{4}=-\frac{\delta_{v} f_{\overline{2}}}{\left\|\delta_{v} f_{\overline{2}}\right\|} .
$$

The angles between the edges emanating from $f$ are denoted by $\alpha_{12}=\angle\left(e_{1}, e_{2}\right)=\arccos \left\langle e_{1}, e_{2}\right\rangle, \quad \alpha_{\overline{1} 2}=\angle\left(e_{2}, e_{3}\right)=\arccos \left\langle e_{2}, e_{3}\right\rangle$, $\alpha_{\overline{1} \overline{2}}=\angle\left(e_{3}, e_{4}\right)=\arccos \left\langle e_{3}, e_{4}\right\rangle, \quad \alpha_{1 \overline{2}}=\angle\left(e_{4}, e_{1}\right)=\arccos \left\langle e_{4}, e_{1}\right\rangle$,

where $\langle\cdot, \cdot\rangle$ denotes the Euclidean scalar product.

We define the unit tangent vectors in $u$ - and $v$-direction as the normalized sum of the two consecutive unit edge vectors in these directions

$$
T^{u}=\frac{e_{1}-e_{3}}{\left\|e_{1}-e_{3}\right\|} \quad \text { and } \quad T^{v}=\frac{e_{2}-e_{4}}{\left\|e_{2}-e_{4}\right\|} .
$$

Further, we define the unit length principal normals of the parameter polylines in $u$-and $v$-direction at vertices $f$ where the angle between consecutive polylines segments is not 0 or $\pi$ by

$$
N^{u}=\frac{e_{1}+e_{3}}{\left\|e_{1}+e_{3}\right\|} \quad \text { and } \quad N^{v}=\frac{e_{2}+e_{4}}{\left\|e_{2}+e_{4}\right\|} .
$$

The unit tangent vector and the unit principal normal vector are illustrated in the osculating plane in Figure 5. In the above chosen discretization, the tangent vector and the principal normal vector are orthogonal, i.e., $N^{u} \perp T^{u}$ and $N^{v} \perp T^{v}$.

With this basis of the tangent plane $\left(T^{u}, T^{v}\right)$ we can consistently define the unit normal vector of the net at a vertex $f$ by

$$
N=\frac{T^{u} \times T^{v}}{\left\|T^{u} \times T^{v}\right\|} .
$$

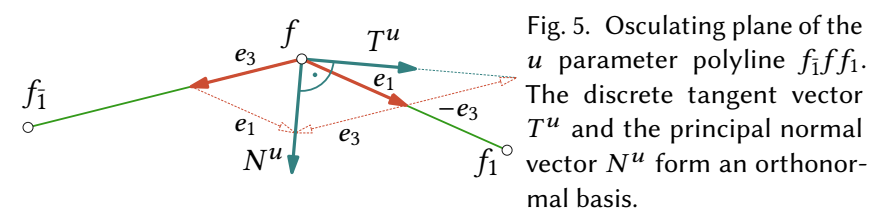

2.2.2 Discrete geodesic parameter lines. The following definition is a discrete version of the well known fact that a curve on a surface is a geodesic if and only if its principal normal coincides (up to orientation) with the surface normal.

Definition 2.2. A $u$-parameter line is a discrete geodesic if and only if $N^{u}$ is parallel to $N$.

Note that the notion of a discrete geodesic is only defined for interior polylines and not for boundary curves.

Lemma 2.3. A discrete u-parameter line is a discrete geodesic if and only if at each vertex $f$ the sum of cosines of angles between emanating edges on both sides of the u-parameter line are equal, i.e.,

$$
\cos \alpha_{12}+\cos \alpha_{\overline{1} 2}=\cos \alpha_{1 \overline{2}}+\cos \alpha_{\overline{1} \overline{2}} \text {. }
$$

Proof. First, let us recall from above that $N^{u}$ is always orthogonal to $T^{u}$. Then, we have

$$
\begin{aligned}
N^{u} \| N & \Leftrightarrow N^{u} \perp T^{u}, T^{v} \Leftrightarrow N^{u} \perp T^{v} \Leftrightarrow\left\langle e_{1}+e_{3}, e_{2}-e_{4}\right\rangle=0 \\
& \Leftrightarrow\left\langle e_{1}, e_{2}\right\rangle+\left\langle e_{2}, e_{3}\right\rangle-\left\langle e_{1}, e_{4}\right\rangle-\left\langle e_{3}, e_{4}\right\rangle=0 \\
& \Leftrightarrow \cos \alpha_{12}+\cos \alpha_{\overline{1} 2}-\cos \alpha_{1 \overline{2}}-\cos \alpha_{\overline{1} \overline{2}}=0 .
\end{aligned}
$$

2.2.3 Discrete orthogonal nets. Having tangents for the parameter lines, it is most natural to define orthogonality of a net as follows:

Definition 2.4. A discrete net is a discrete orthogonal net if and only if at each vertex the tangent vectors $T^{u}$ and $T^{v}$ of the parameter lines are orthogonal.

LEMMA 2.5. A discrete net is a discrete orthogonal net if and only if at each vertex the sums of cosines of opposite angles are equal, i.e., if

$$
\cos \alpha_{12}+\cos \alpha_{\overline{1} \overline{2}}=\cos \alpha_{1 \overline{2}}+\cos \alpha_{\overline{1} 2} .
$$

Proof. We have

$$
\begin{aligned}
T^{u} \perp T^{v} & \Leftrightarrow\left\langle e_{1}-e_{3}, e_{2}-e_{4}\right\rangle=0 \\
& \Leftrightarrow\left\langle e_{1}, e_{2}\right\rangle-\left\langle e_{2}, e_{3}\right\rangle-\left\langle e_{1}, e_{4}\right\rangle+\left\langle e_{3}, e_{4}\right\rangle=0 \\
& \Leftrightarrow \cos \alpha_{12}-\cos \alpha_{\overline{1} 2}-\cos \alpha_{1 \overline{2}}+\cos \alpha_{\overline{1} \overline{2}}=0 .
\end{aligned}
$$

Remark. Another definition of discrete geodesics, namely $\alpha_{12}+$ $\alpha_{\overline{1} 2}=\alpha_{1 \overline{2}}+\alpha_{\overline{1} \overline{2}}$, has been employed previously in connection with polyhedral surfaces [Polthier and Schmies 1998; Wunderlich 1951]. Similarly, a version of discrete orthogonality without the cosines $\left(\alpha_{12}+\alpha_{\overline{1}}=\alpha_{1 \overline{2}}+\alpha_{\overline{1} 2}\right)$ has been used for polyhedral surfaces, namely 
in the theory of conical nets [Liu et al. 2006]. The presence of the cosines in the characterizing equations of Lemmas 2.3 and 2.5 is an advantage for numerical optimization, since they are bilinear in variables (edge unit vectors) which one needs in the optimization anyway. Hence they fit the proposal by [Tang et al. 2014] to accelerate the convergence of Gauss-Newton methods through the use of at most quadratic constraints, even at the price of introducing additional variables.

The following definition is essential for our paper. It discretizes the notion of geodesic parallel coordinates.

Definition 2.6. A discrete net is parametrized by discrete geodesic parallel coordinates if the net is discrete orthogonal and the $u$ parameter lines are discrete geodesics.

Proposition 2.7. The following three are equivalent:

(i) $f$ is parametrized by discrete geodesic parallel coordinates.

(ii) At every vertex $f$ we have $\alpha_{12}=\alpha_{12}$ and $\alpha_{\overline{1} 2}=\alpha_{\overline{1}}$ (Fig. 6 left).

(iii) The discrete osculating plane of the $u$-lines (spanned by $f_{\overline{1}}, f, f_{1}$ ) at each vertex $f$ is the bisector plane of the $v$-line edges, i.e., $e_{2}$ and $e_{4}$ are mirror-symmetric with respect to the osculating plane in u-direction (see Figure 6 right).

Proof. The equivalence of (i) and (ii) is clear after adding and subtracting the characterizing equations from Lemmas 2.3 and 2.5 which yields $\cos \alpha_{12}=\cos \alpha_{12}$ and $\cos \alpha_{\overline{1} 2}=\cos \alpha_{\overline{1} \overline{2}}$, and therefore equality of the angles.

The equivalence of (ii) and (iii) follows by angle preservation of reflections (cf. Figure 6 right). Mirror symmetry of $e_{2}$ and $e_{4}$ with a symmetry plane containing $e_{1}$ implies equal angles $\alpha_{12}=\alpha_{12}$ and analogously $\alpha_{\overline{1} 2}=\alpha_{\overline{1} \overline{2}}$.

On the other hand, equality of the angles $\alpha_{12}=\alpha_{1 \overline{2}}$ implies that $e_{2}$ and $e_{4}$ are rulings of a right circular cone with axis $f f_{1}$ and vertex $f$. Analogously, $e_{2}$ and $e_{4}$ are rulings of a right circular cone with axis $f f_{\overline{1}}$ and the same vertex $f$. As both cones are symmetric with respect to the osculating plane $f f_{1} f_{\overline{1}}$, the vectors $e_{2}, e_{4}$ are mirror-symmetric to the same plane.

Note, that (iii) only makes sense if the three vertices $f_{\overline{1}}, f, f_{1}$ do not lie on a common straight line. In that case the equivalence statement only holds for (i) and (ii).
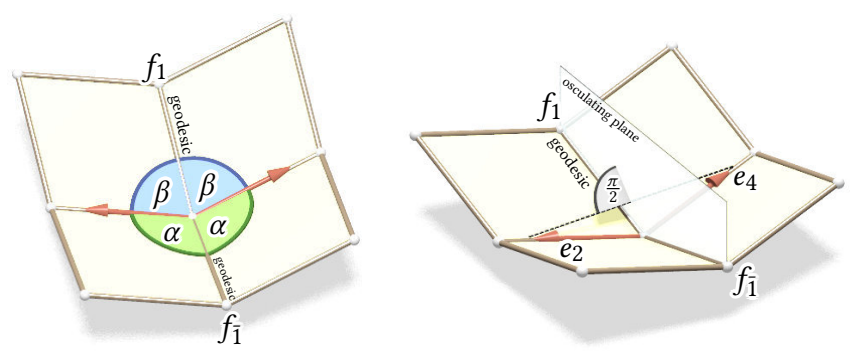

Fig. 6. The geometric characterizations of discrete geodesic parallel coordinates as in Proposition 2.7. Left: Angle condition. Any two neighboring angles "symmetric" to the path of the geodesic are equal. Right: The unit length edge vectors transversal to the geodesic are mirror symmetric with respect to the osculating plane $f_{\overline{1}} f f_{1}$.

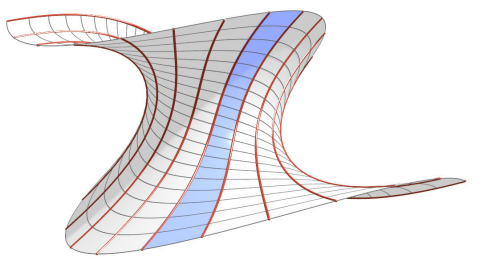

Fig. 7. By a geodesic strip we understand the surface between two geodesics of a parametrization by geodesic parallel coordinates.

\section{GEODESIC STRIP MODELS}

Discrete geodesic parallel coordinates divide a surface into geodesic strips. Here we discuss how the variation of the strip width across a strip is related to the Gaussian curvature and show how to control the width in order to model nearly developable surfaces.

\subsection{Geodesic strips and strip width}

We call the surface strip between two geodesics of a parametrization by geodesic parallel coordinates a geodesic strip (see Figure 7). Let us again assume that the $u$-lines represent the family of the geodesics and the $v$-lines constitute the orthogonal family of parameter lines. Then we quantify the strip width of a geodesic strip with the function that measures the lengths of the $v$-parameter lines between the boundary geodesics of that strip. Therefore, the strip width between two $\varepsilon$-close geodesics is

$$
\int_{v}^{v+\varepsilon}\left\|f_{v}(u, t)\right\| d t \approx \varepsilon\left\|f_{v}(u, v)\right\|+\varepsilon^{2}(\ldots) .
$$

Consequently, the first order approximation of the strip width is the length of the derivative of the $v$-lines: $\left\|f_{v}\right\|$. Assuming arc length parametrization in the $u$-direction (i.e., $\left\|f_{u}\right\|=1$ ), which is always possible for geodesic parallel coordinates (see e.g., [Kühnel 2003, p. 110]), the strip width is closely related to the Gaussian curvature $K$ via a linear ODE, the well known Jacobi differential equation (cf. [Kühnel 2003])

$$
\partial_{u u} w(u)+w(u) K=0,
$$

where $w(u)$ is the infinitesimal strip width. We will utilize this Jacobi differential equation in our discrete model of nearly developable surfaces.

\subsection{Nearly developable surfaces}

A developable surface is a surface which is isometric to the plane. Such surfaces are also characterized by everywhere vanishing Gaussian curvature $(K=0)$. Since the Gaussian curvature scales by a factor of $\frac{1}{\lambda^{2}}$ when the surface is scaled by $\lambda$, nearly developable surfaces must be considered as surfaces where the Gaussian curvature is small compared to the size of that surface. Even though mathematically this notion is rather vague, application-wise it is of importance, as some materials like leather or felt allow for some stretching or shrinking while being deformed. Our reasoning is underpinned by the theorem of Bertrand and Puiseux [Spivak 1979] for the Gaussian curvature $K$ which basically says that $K$ is measured by the limit of the difference between the area of a geodesic circle on the surface and the area of a circle in the plane. If $K$ is close to zero everywhere then the area of the surface is everywhere close to the area of the plane. See Figure 8 for an example of a nearly developable surface.

In the case of vanishing Gaussian curvature the Jacobi differential equation (1) simplifies to $\partial_{u u}\left(\left\|f_{v}\right\|\right)=0$. Under the assumption of a 
discrete arc length parametrization in $u$-direction, discretizing this Jacobi equation straightforwardly yields

$$
\delta_{u u}\left(\left\|\delta_{v} f_{\overline{1}}\right\|\right)=0
$$

which gives

$$
\left\|f_{12}-f_{1}\right\|-2\left\|f_{2}-f\right\|+\left\|f_{\overline{1} 2}-f_{\overline{1}}\right\|=0 .
$$

In our optimization algorithm, the edge lengths become variables and this discrete Jacobi equation is one of the constraints for nearly developable surfaces (cf. Eqn. (8)).
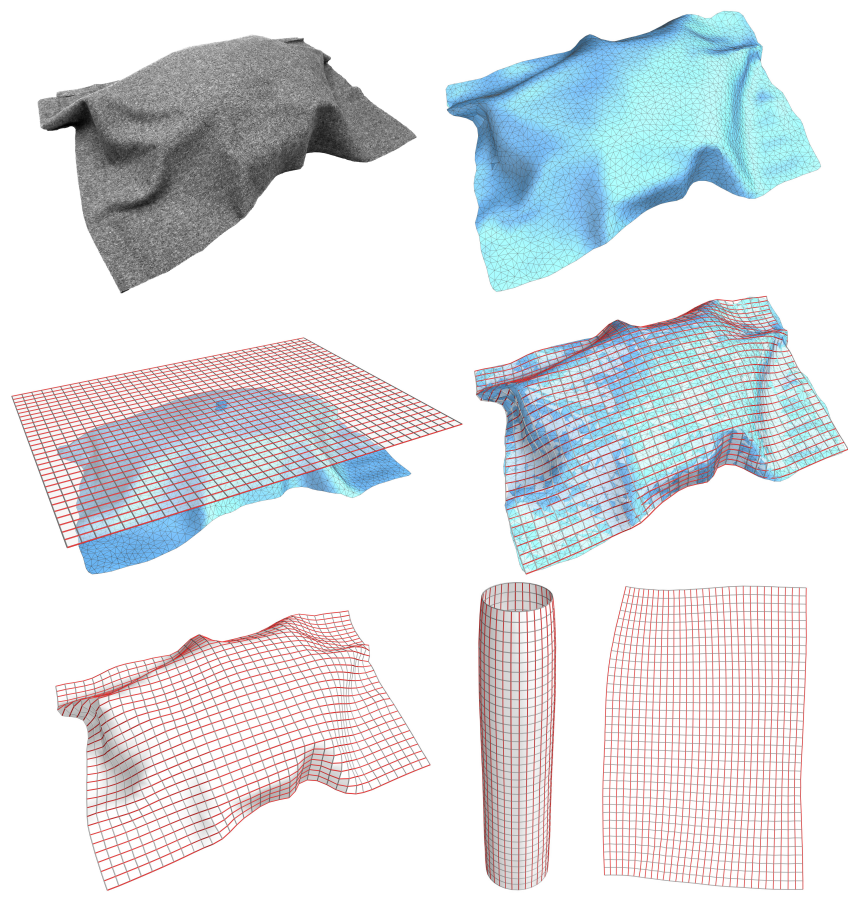

Fig. 8. Nearly developable surface. We scanned a 3D shape modeled from a piece of felt (top-left) and extracted a triangle mesh out of its point cloud (top-right). The triangle mesh has been approximated by discrete nearly developable surfaces with a discrete geodesic parallel coordinate net (centerright and bottom-left). For this approximation task we need a sensible initial guess of the resulting surface (center-left). The discrete model and the given triangle mesh fit together very well as confirmed by a Hausdorff distance of 0.0111 between the reference mesh (with bounding box diagonal length 46.1) and our nearly developable mesh (overlay of both meshes center-right). The corresponding isometric surface of revolution looks almost developable (i.e., like a right circular cylinder; bottom-center). The nearly developable surface has been developed into the plane (bottom-right).

\section{SURFACES ISOMETRIC TO SURFACES OF REVOLUTION}

Let us recall that one of our main goals is to model intrinsically symmetric surfaces. They shall be isometrically deformable into themselves, like a rotational surface can be moved into itself. That is why we are interested in surfaces which are isometric to surfaces of revolution. Since isometric deformations of surfaces transform geodesics to geodesics and they do not change any intersection angles between curves on the surfaces, it is sensible to consider isometric deformations within the framework of geodesic parallel coordinates. Thus, we now derive criteria under which geodesic parallel coordinates describe surfaces that are isometric to surfaces of revolution.

\subsection{Smooth surfaces isometric to surfaces of revolution}

Before we consider the discrete setting let us first characterize surfaces which are isometric to surfaces of revolution in terms of geodesic parallel coordinates (Proposition 4.2). Before we get there we have to consider the following technical lemma.

LemMA 4.1 ([KüHNEL 2003, P. 110]). Let $f$ be a surface parametrized by geodesic parallel coordinates. Then $\partial_{v}\left\|f_{u}(u, v)\right\|=0$, i.e., $\left\|f_{u}(u, v)\right\|$ does not depend on $v$.

Proof. The geodesic curvature $\kappa_{g}^{u}$ [Kühnel 2003] of the $u$-parameter lines $(v=$ const) of an orthogonal net is

$$
\kappa_{g}^{u}=-\frac{\partial_{v} E}{2 E \sqrt{G}}
$$

where $E, F, G$ are the coordinate functions of the first fundamental form, i.e., $E=\left\langle f_{u}, f_{u}\right\rangle$, etc. In our case the $u$-parameter lines are geodesics which implies that the geodesic curvature vanishes $\kappa_{g}^{u}=$ 0 . Consequently, $0=\partial_{v} E=\partial_{v}\left\|f_{u}(u, v)\right\|^{2}$ which implies that $\left\|f_{u}(u, v)\right\|$ does not depend on $v$.

Let $r:\left[u_{0}, u_{1}\right] \rightarrow \mathbb{R}$ be any function. Then, by Lemma 4.1 the following function only depends on $u$.

$$
h(u):=\int_{u_{0}}^{u} \sqrt{\left\|f_{u}(t, v)\right\|^{2}-r^{\prime}(t)^{2}} d t .
$$

Proposition 4.2. Let $f$ be a surface parametrized by geodesic parallel coordinates with the additional property that for any $a \in$ $\left[u_{0}, u_{1}\right]$

$$
\frac{\left\|f_{v}(u, v)\right\|}{\left\|f_{v}(a, v)\right\|}=: r(u)
$$

only depends on $u$ (see the illustration in Figure 9 left). Then $f$ is isometric to the surface of revolution $\tilde{f}$ with

$$
\tilde{f}=(r(u) \cos \phi(v), r(u) \sin \phi(v), h(u)),
$$

where $\phi(v):=\int_{v_{0}}^{v}\left\|f_{v}(a, s)\right\| d s$ and where $h$ is defined as in (3) with $r$ from (4).

Proof. We have

$$
\tilde{f}_{u}=\left(r^{\prime} \cos \phi, r^{\prime} \sin \phi, h^{\prime}\right)=\left(r^{\prime} \cos \phi, r^{\prime} \sin \phi, \sqrt{\left\|f_{u}\right\|^{2}-r^{\prime 2}}\right),
$$

and

$$
\tilde{f}_{v}=\left(-r \phi^{\prime} \sin \phi, r \phi^{\prime} \cos \phi, 0\right),
$$

and therefore $\tilde{E}=\left\|\tilde{f}_{u}\right\|^{2}=r^{\prime 2}+\left\|f_{u}\right\|^{2}-r^{\prime 2}=\left\|f_{u}\right\|^{2}=E$ and $\tilde{G}=\left\|\tilde{f}_{\mathcal{v}}\right\|^{2}=r^{2} \phi^{\prime 2}=\frac{\left\|f_{v}(u, v)\right\|^{2}}{\left\|f_{v}(a, v)\right\|^{2}}\left\|f_{v}(a, v)\right\|^{2}=\left\|f_{v}(u, v)\right\|^{2}=G$. Cosequently, $\tilde{E}=E, \tilde{F}=F=0, \tilde{G}=G$, which implies isometry. 

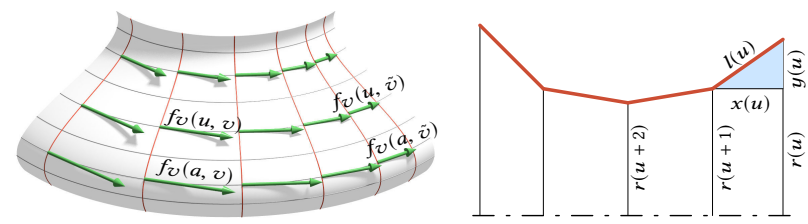

Fig. 9. Left: On surfaces which are isometric to surfaces of revolution the ratios of the lengths of the derivatives in $v$ direction (i.e., in the rotation direction) is independent of $v$, i.e., $\frac{\left\|f_{v}(u, v)\right\|}{\left\|f_{v}(a, v)\right\|}=r(u)$ (see Proposition 4.2) Right: Profile of the discrete surface of revolution with the discrete meridian curve (red). $y(u)$ is the difference of consecutive radii $r(u+1)-r(u)$.

\subsection{Discrete surfaces isometric to surfaces of revolution}

To obtain a discrete surface which is isometric to a surface of revolution we can take a net parametrized by discrete parallel geodesic coordinates and "add" a discrete version of the required property stated in Proposition 4.2. For that we reconsider the independence of the term (4) from $v$ which is equivalent to

$$
\frac{\left\|f_{v}(a, v)\right\|}{\left\|f_{v}(b, v)\right\|}
$$

being independent of $v$ for any $a, b \in\left[u_{0}, u_{1}\right]$. We discretize this property straightforwardly by requiring for any integers $a, b$ in the domain of definition

$$
\frac{\left\|\delta_{v} f(a, v)\right\|}{\left\|\delta_{v} f(b, v)\right\|}=\text { const, }
$$

for all integers $v$. With that we arrive at a characterization of a discrete net to be discrete isometric to a surface of revolution (in its common parametrization), namely if it is parametrized by discrete geodesic parallel coordinates and fulfills (5).

Note, that our notion of surfaces which are isometric to a surface of revolution does not involve an actual surface of revolution to which it is isometric. However, for some applications it might be desirable or necessary to compute the corresponding surface of revolution. We will attend to that matter below (§ 4.2.2).

4.2.1 Intrinsic repetitivity. The advantage in cost- and effort-reduction is obvious when cladding surfaces repetitively with the same surface strip. Thus, for practical applications it makes sense to require constant edge lengths in each row of geodesic parallels, i.e., for any integer $a,\left\|\delta_{v} f(a, v)\right\|$ is required to be the same for all integers $v$. This automatically implies (5) and therefore isometry to a surface of revolution.

4.2.2 Extracting the surface of revolution. Once we have found a discrete net which is isometric to a surface of revolution with the properties of $\S 4.2 .1$ by optimization (see Section 5 ) we can compute the surface of revolution, to which it is actually isometric, in the following way.

Let us assume that the rectangular patch has $m \times n$ vertices with indices $[1, m] \times[1, n]$, with the $m$ vertices in the $u$ direction, i.e., in the direction of the geodesic. Then for $u \in[1, m]$ we compute the length of the average edge length of the regular $(n-1)$-gons corresponding to the parallel circles of the surface of revolution

$$
d(u):=\frac{1}{n-1} \sum_{i=1}^{n-1}\left\|\delta_{v} f(u, i)\right\| .
$$
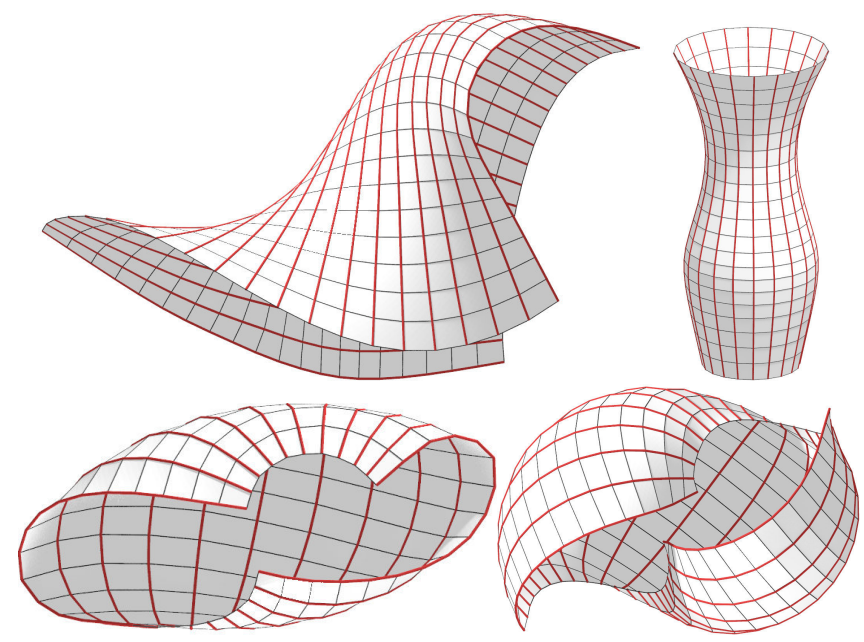

Fig. 10. A net parametrized by discrete geodesic parallel coordinates (topleft) which is isometric to a surface of revolution (top-right). Bottom-row: Isometric deformation of a half sphere (left) and a sphere with its "polar caps" removed (right). Due to Gauss' theorema egregium, these are surfaces of positive constant Gaussian curvature.

Consequently, the radius of the circumcircle of such an $(n-1)$-gon is $r(u):=\frac{d(u)}{2 \sin (\pi /(n-1))}$. Here, we assume that the isometric surface of revolution makes a full rotation about its axis. This condition can be relaxed (see below; Prop. 4.3). Further, for $u \in[1, m-1]$ we compute the average length of the polygon segment on the meridian curve

$$
l(u):=\frac{1}{n} \sum_{i=1}^{n}\left\|\delta_{u} f(u, i)\right\| .
$$

With this data we can construct the discrete reference surface of revolution. As illustrated by Figure 9 (right) we compute the height differences between two parallel circles by

$$
x(u):=\sqrt{l(u)^{2}-(r(u+1)-r(u))^{2}} .
$$

Then, the discrete surface of revolution is parametrized by

$$
f(u, v)=(r(u) \cos (2 v \pi /(n-1)), r(u) \sin (2 v \pi /(n-1)), h(u)),
$$

where $h(u)=\sum_{i=1}^{u} x(i)$. Instead of transforming the entire rectangular surface patch to a surface of revolution of exactly one full rotation, we could do the same procedure just for some sub-patch as would be also possible in the smooth case due to the following theorem.

Proposition 4.3 (Theorem of Minding [1838]). Let $f(u, v)=$ $(r(u) \cos v, r(u) \sin v, h(u))$ be a surface of revolution. Then for any $\lambda \in \mathbb{R}$ for which the integral

$$
\tilde{h}(u):=\int_{u_{0}}^{u} \sqrt{\left(1-\lambda^{2}\right) r^{\prime}(t)^{2}+h^{\prime}(t)^{2}} d t
$$

is real (which is always the case for $\lambda \in[0,1])$, the surface of revolution

$$
f^{\lambda}(u, v):=\left(\lambda r(u) \cos \frac{v}{\lambda}, \lambda r(u) \sin \frac{v}{\lambda}, \tilde{h}(u)\right)
$$

is isometric to $f$. Note, $f^{1}=f$. 


\section{ALGORITHM}

We model our discrete surfaces by defining a constraint manifold in the space of all variables, choose a starting point and try to "move" towards fulfilling the constraints. Different constraints are multiplied with different weights. A crucial point in the optimization lies in choosing the weights in sensible ways. Tang et al. [2014] proposed such a method, which is a Gauss-Newton variant, and made the observation that it works best if the constraints are formulated as linear or quadratic functions. Some constraints which are a priori not quadratic can sometimes be reformulated into quadratic terms with the help of additional variables which then become subject to optimization themselves. In the following, we describe a list of constraints which, in different combinations and with different weights, describe the geometry that we are looking for.

\subsection{List of constraints}

List of variables. We are working with meshes $\mathcal{M}=(V, E, F)$ with the combinatorics of an $(m \times n)$ rectangular sub-patch of the $\mathbb{Z}^{2}$ lattice. Thus, a general mesh has $|V|=n m$ vertices, $|F|=(m-$ 1) $(n-1)$ faces, and $|E|=n(m-1)+m(n-1)$ edges. The variables in our system are:

\begin{tabular}{|l|l|l|}
\hline variables for & notation & number \\
\hline coordinates of vertices & $f_{i} \in \mathbb{R}^{3}$ & $3|V|$ \\
\hline components of (unit) edge vectors & $e_{i j}, e_{j i} \in \mathbb{R}^{3}$ & $6|E|$ \\
\hline edge lengths & $w_{i j}, w_{j i} \in \mathbb{R}$ & $2|E|$ \\
\hline
\end{tabular}

Consequently, these are in total $8|E|+3|V|$ variables. In the following we list our constraints and put the number of the considered equations in square brackets. Since our discrete geodesic parallel coordinates are nets where both families of parameter lines play different roles, we cannot express all constraints independently from the coordinate direction.

Net constraints. As mentioned before we use more variables than actually necessary to describe our nets but obtain, as a consequence, algebraic and at most quadratic equations as constraints. Consequently, to describe a proper net we need to tie the edge length variables $w_{i j}, w_{j i}$ to the actual edges:

$$
\left(f_{i}-f_{j}\right)-w_{i j} e_{i j}=0, \quad\left(f_{j}-f_{i}\right)-w_{j i} e_{j i}=0,
$$

and

$$
\left(f_{i}-f_{j}\right)^{2}-w_{i j}^{2}=0, \quad\left(f_{j}-f_{i}\right)^{2}-w_{j i}^{2}=0 .
$$

These two sets of constraints imply that the vectors $e_{i j}$ and $e_{j i}$ are unit length: i.e., $e_{i j}^{2}=e_{j i}^{2}-1=0$. The theoretical appearance of negative widths $w_{i j}$ is remedied by the use of fairness terms (see below) which prevent unwanted flipping of $e_{i j}$ directions and sign changes of $w_{i j}$. Otherwise the corresponding constraint could be implemented easily as $w_{i j}-d_{i j}^{2}=0$ for some extra variables $d_{i j} \in \mathbb{R}$.

Discrete geodesic parallel coordinate constraints. This constraint can be formulated as an angle condition, cf. Proposition 2.7 (ii) or equivalently as a condition of the cosine (cf. remark after Lemma 2.5). For a vertex star with central vertex $f_{i}$ and edge-adjacent vertices $f_{j}, f_{k}, f_{l}, f_{m}$ with $f_{l}, f_{i}, f_{j}$ being the discrete geodesic, the angle constraint reads

$$
\left\langle e_{i j}, e_{i k}\right\rangle-\left\langle e_{i m}, e_{i j}\right\rangle=0, \quad\left\langle e_{i l}, e_{i k}\right\rangle-\left\langle e_{i m}, e_{i l}\right\rangle=0 .
$$

Discrete arc length parametrization in $u$ direction. In geodesic parallel coordinates, all the $u$ parameter lines can be parametrized proportional to arc length simultaneously. Thus, we constrain consecutive edges in $u$ direction to have equal lengths:

$$
w_{i j}-w_{l i}=0, \quad w_{j i}-w_{i l}=0 .
$$

Equal strip width function. As it is one goal of our paper to obtain intrinsic repetitivity, i.e., cladding a surface just with a sequence of strips that are isometric to each other (cf. Figure 2), we want the strips to be of equal lengths and to have an equal distribution of the strip width along each strip. To get that we have to constrain the lengths of consecutive edges along the orthogonal parameter lines ( $v$ direction) to be of equal lengths:

$$
w_{i k}-w_{i m}=0, \quad w_{k i}-w_{m i}=0 .
$$

Note that we obtain strips of equal lengths automatically. This is a property of geodesic parallel coordinates (cf. [Kühnel 2003, Def. 4.27]) and it carries over to our discretization so well that we do not even switch on corresponding constraints similar to the ones above.

Nearly developable constraints. For nearly developable surfaces we just formulate the simplified Jacobi equation (2) for $K=0$ as a constraint. The second difference in $u$ direction of edge lengths in $v$ direction reads

$$
\delta_{u u} w_{i j}=w_{i-1, j}-2 w_{i j}+w_{i+1, j}=0, \quad \delta_{u u} w_{j i}=0 .
$$

Note that the Jacobi equation as we use it here requires arc length parametrization in $u$ direction. Consequently, we additionally have to enforce the corresponding constraint from above.

Isometry constraints. Let us denote by $w_{i j}^{0}$ the edge lengths of a given mesh to which we compute the isometry. Then

$$
w_{i j}-w_{i j}^{0}=0, \quad w_{j i}-w_{j i}^{0}=0 .
$$

Approximation constraints. Given a reference surface with vertices $p_{k}$ and normal vectors $n_{k}$. For each vertex $f_{i}$ of our net let $p_{k_{i}}$ be the closest point of the reference surface to $f_{i}$.

$$
\left\langle f_{i}-p_{k_{i}}, n_{k_{i}}\right\rangle=0 .
$$

The closest points are computed in every iteration of the GaussNewton step.

Constraints for handle-based deformations. We prescribe $l$ vertex positions $p_{k}$. Then

$$
f_{i}-p_{k_{i}}=0 .
$$

Fairness constraints. To obtain good mesh quality as an optimization result we require some fairness terms. The constraints are realized by vanishing second forward differences:

$$
f_{l}-2 f_{i}+f_{j}=0, \quad f_{k}-2 f_{i}+f_{m}=0 .
$$

The following table collects the number of constraints and the resulting degrees of freedom for each individual constraint. 


\begin{tabular}{|l|c|c|}
\hline constraint & no. of constraints & dof \\
\hline \hline net constraints & $8|E|$ & $3|V|$ \\
\hline disc. geod. par. coord. & $2(m n-n-2 m+2)$ & $m n+2 n+4 m-4$ \\
\hline arc length $u$-direction & $2 n(m-2)$ & $m n+4 n$ \\
\hline equal strip widths & $2 m(n-2)$ & $m n+4 m$ \\
\hline nearly developable & $2(2 n-1)(m-2)$ & $8 n+2 m-m n-4$ \\
\hline isometry & $2|E|$ & $m n+m+n$ \\
\hline approximation & $|V|$ & $2 m n$ \\
\hline handle based def. & $3 l$ & $3 m n-3 l$ \\
\hline fairness & $2(m n-n-m)$ & $m n+2 n+2 m$ \\
\hline
\end{tabular}

As a constraint solver for our algorithm we use the guided projection algorithm of [Tang et al. 2014].

\subsection{Computation times}

The computation times $T$ in the following table refer to our implementation in Python on an Intel ${ }^{\circledR}$ Core $^{\mathrm{TM}}$ i5-6260U CPU with $1.80 \mathrm{GHz}, 8 \mathrm{~GB}$ RAM. The times are measured in seconds per iteration as the modeling procedure is interactive on a handle-based editing method. While we drag a vertex with the mouse, we constantly run iterations of our algorithm until the mouse button is released, and after that five more iterations follow. The weights of the constraints are set to 1 or 0 except for the weights of the fairness terms. The small arrows (e.g., .01 $\rightarrow 0$ ) mean that we lower the corresponding weights to zero during our editing process if we don't expect any severe changes in the geometry by releasing the corresponding constraint. The following table also shows which constraints are switched on in the optimization.

\begin{tabular}{|c|c|c||c|c|c|c|c|c|c||c|}
\hline Fig. & $|\mathrm{V}|$ & $|\operatorname{var}|$ & $w_{\text {go }}$ & $w_{\text {iso }}$ & $w_{\text {esw }}$ & $w_{\text {al }}$ & $w_{\text {dev }}$ & $w_{\text {prox }}$ & $w_{\text {fair }}$ & $T /$ iter \\
\hline \hline 1 & 441 & 8043 & 1 & 1 & 0 & 0 & 0 & 0 & $.01 \rightarrow 0$ & 0.25 \\
\hline 2 & 391 & 742 & 1 & 1 & 0 & 0 & 0 & 0 & $.01 \rightarrow 0$ & 0.11 \\
\hline 8 & 1147 & 21249 & 1 & 0 & 1 & 1 & 1 & 1 & $.1 \rightarrow .01$ & 1.43 \\
\hline $10-1$ & 441 & 8043 & 1 & 1 & 0 & 0 & 0 & 0 & $.01 \rightarrow 0$ & 0.17 \\
\hline $10-2$ & 187 & 3329 & 1 & 1 & 0 & 0 & 0 & 0 & 0 & 0.07 \\
\hline $11-1$ & 330 & 5942 & 1 & $1 \rightarrow 0$ & $1 \rightarrow 0$ & 0 & 0 & 1 & - & 0.23 \\
\hline $11-2$ & 4797 & 89879 & 1 & $1 \rightarrow 0$ & $1 \rightarrow 0$ & 0 & 0 & 1 & - & 6.62 \\
\hline$? ?$ & 1458 & 27054 & 1 & 0 & $1 \rightarrow 0$ & 0 & 0 & 1 & $.1 \rightarrow 0$ & 1.38 \\
\hline 15 & 441 & 8043 & 1 & 0 & 1 & 1 & 1 & 0 & .001 & 0.43 \\
\hline
\end{tabular}

The weights in the above table control the following: $w_{\mathrm{go}}=$ discrete geodesic parallel coordinates; $w_{\text {iso }}=$ isometry to reference surface; $w_{\text {esw }}=$ equal strip widths; $w_{\text {al }}=$ arc length parametrization in $u$ direction; $w_{\mathrm{dev}}=$ near developability; $w_{\text {prox }}=$ closeness to reference surface; $w_{\text {fair }}=$ fairness.

\section{RESULTS AND APPLICATIONS}

\subsection{Verification}

Clairaut's relation. To verify the quality of our geodesics we approximate a surface of revolution by nontrivial discrete geodesic parallel coordinates and compare it with Clairaut's relation. It says that any geodesic on a surface of revolution fulfills

$$
r(t) \cos \alpha(t)=\text { const, }
$$

where $r(t)$ is the distance of the geodesic to the axis of revolution and $\alpha(t)$ measures the angle between the geodesic and the latitude. In our discrete model (Figure 11) we compute $r_{i} \cos \alpha_{i}$ along each

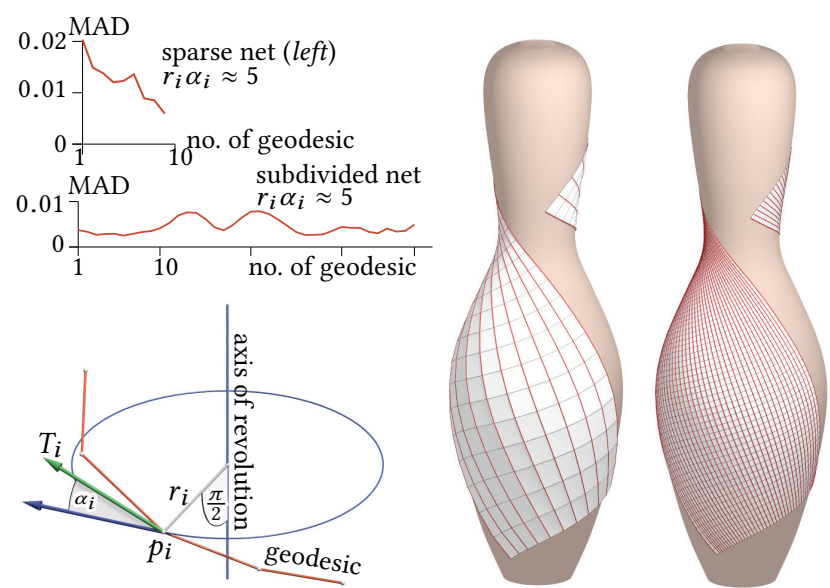

Fig. 11. We lay a net of discrete geodesic parallel coordinates over a surface of revolution in such a way, that the geodesics (red) do not correspond to the meridian curves but are rather twisted compared to them (left bowling pin). After subdividing the net and optimizing again we obtain denser discrete geodesic parallel coordinates (right bowling pin). We measure $r_{i} \cos \alpha_{i}$ (cf. bottom-left) for each vertex which, according to Clairaut's relation (cf. Eqn. (9)), should be constant (within tolerances) along each discrete geodesic. With an average value of $r_{i} \cos \alpha_{i} \approx 5$ the mean absolute deviation (MAD) is at most 0.02 for the sparse net and the errors go down even more as we subdivide and optimize the mesh to under 0.01 (cf. diagrams top-left).

geodesic line of the net, where $r_{i}$ is the distance of vertex $p_{i}$ to the axis of revolution and where $\alpha_{i}$ is the angle between the tangent vector $T_{i}$ and the parallel circle. It turns out that these values are constant within reasonable tolerances as depicted in Figure 11. We have no rigorous proof that the discrete geodesics converge to their smooth counterparts after subdivision and re-optimization, but we have strong numerical evidence that the errors go down.

Ruling vector field of developable surfaces. Developable surfaces are ruled surfaces and thus have a ruling vector field which is unique in non-flat areas. Viewing rulings as intersections of neighboring tangent planes gives a simple way to compute the direction field by simply computing the cross product of neighboring normal vectors $N \times N_{1}$ or $N \times N_{2}$. Figure 12 nicely illustrates the vector field of ruling directions of a nearly developable surface.

Measure of isometry. We measure the isometry between an original surface $f^{0}$ and a deformed surface $f$ with the well known two dimensional strain tensor from solid mechanics [Sadd 2009]. The strain matrix adapted for our discrete parallel geodesic coordinates is defined at vertices and formulated in the basis of tangent vectors $T_{1}, T_{2}$. It reads

$$
D=\left(\begin{array}{cc}
\frac{\varepsilon\left(l_{1}\right)+\varepsilon\left(l_{\overline{1}}\right)}{2} & \tan \left(\alpha_{\overline{1} \overline{2}}-\alpha_{\overline{1} 2}\right)-\tan \left(\alpha_{1 \overline{2}}-\alpha_{12}\right) \\
\tan \left(\alpha_{\overline{1} \overline{2}}-\alpha_{\overline{1} 2}\right)-\tan \left(\alpha_{1 \overline{2}}-\alpha_{12}\right) & \frac{\varepsilon\left(l_{2}\right)+\varepsilon\left(l_{\overline{2}}\right)}{2}
\end{array}\right),
$$

where $\varepsilon\left(l_{1}\right)=\frac{\left\|\delta_{u} f\right\|-\left\|\delta_{u} f^{0}\right\|}{\left\|\delta_{u} f^{0}\right\|}, \varepsilon\left(l_{\overline{1}}\right)=\frac{\left\|\delta_{u} f_{\overline{1}}\right\|-\left\|\delta_{u} f_{\overline{1}}^{0}\right\|}{\left\|\delta_{u} f_{\overline{1}}^{0}\right\|}$, etc. (for the notation also cf. Figure 4). Let now $\lambda_{1}, \lambda_{2}$ be the eigenvalues and $v_{1}, v_{2}$ the eigenvectors of $D$. The principal distortion directions are $v_{1}^{x} T_{1}+v_{1}^{y} T_{2}$ and $v_{2}^{x} T_{1}+v_{2}^{y} T_{2}$ where $T_{1}, T_{2}$ is the orthogonal basis of the tangent plane aligned with the parameter lines. Note that we 


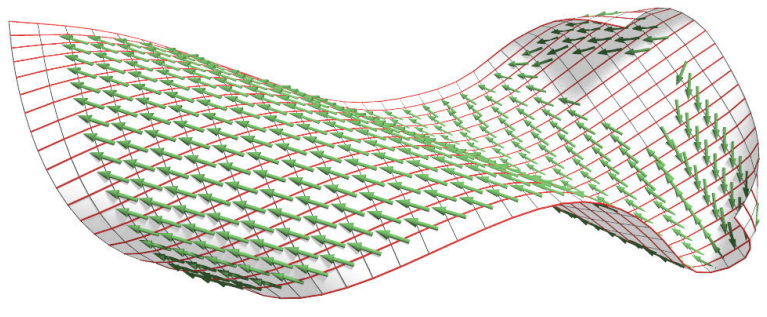

Fig. 12. By requiring (almost) constant strip widths for our geodesic strips we obtain (nearly) developable surfaces. We compute the ruling directions as the cross product of neighboring normal vectors $N \times N_{1}$ or $N \times N_{2}$ wherever possible. Clearly, in flat regions there is no unique ruling direction (as visible in our illustration).
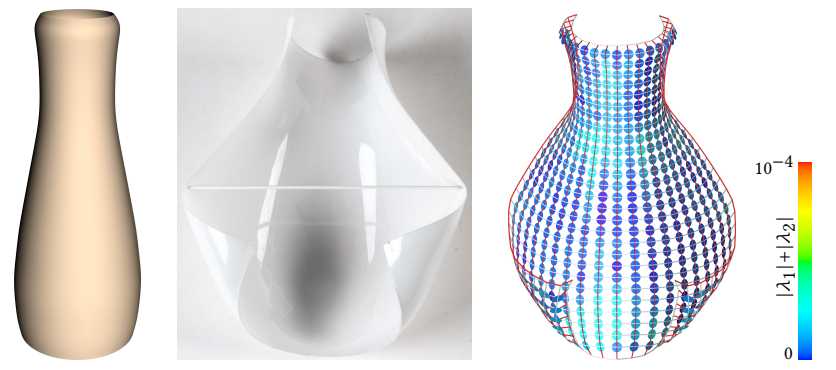

Fig. 13. Isometric deformation of a surface of revolution. (Center) photo of a deformed plastic bottle and (left) image of a 3D digital model of the isometrically same surface of revolution. (Right) illustration of the strain after deformation. The principal directions of the colored ellipses illustrate the distortion directions and the amount of distortion $\left(\left|\lambda_{1}\right|+\left|\lambda_{2}\right|\right)$ is illustrated by the colors. The maximal distortion is 0.00004 .

optimize for discrete orthogonality (cf. Lemma 2.5 and constraint (6)). So we can always assume $T_{1} \perp T_{2}$. A color plot illustration of this isometry measure is depicted in Figure 13.

\subsection{Applications}

Discrete geodesic parallel coordinates very naturally lead to architectural applications in various ways.

6.2.1 Geodesic gridshells. Gridshells are doubly-curved surfaces with favorable static properties and materialized as a grid network. A very basic idea is to simply take elongated quadrilateral timber strips which we imagine to be bent along a thought surface. In that case, these timber strips follow geodesics on that (imaginary) surface as the geodesic curvature, which is invariant under isometries, is vanishing. With our method the design of such surfaces is very simple. We have the means for handle-based design and we can approximate surfaces by discrete geodesic parallel coordinates. For the gridshell in Figure ?? we approximate a given reference surface represented by a triangle net by two transversal nets of discrete geodesic parallel coordinates. Then we just build the two families of geodesics by bending wooden rectangular strips and leave the two families of orthogonal trajectories away.

6.2.2 Nearly developable surfaces. A surface is developable if it can be represented by a family of geodesics which have a constant distance to each other. With our discrete geodesic parallel coordinates we have good access to controlling the strip width function measured along the orthogonal family of parameter lines. Almost constant strip widths imply nearly developable surfaces. While prior work on near developables has been based on a thin Gaussian image [Gavriil et al. 2018], we can also handle shapes as in Fig. 8 whose Gaussian image is not concentrated near a curve.

Cladding. Timber is a material that can be deformed by bending. While thin laths and boards allow for some bending under steady conditions, even thicker pieces of timber allow for surprisingly big deformations, e.g., after heating with water steam. Within this process the timber surfaces undergo deformations which are to a certain degree isometric but also show some stretching and contraction. In Figure 2 we illustrate facade cladding by assembling bent timber strips. The planar development of all strips are congruent. In Figure 15 we illustrate a design from bent timber boards.

Slightly stretchable materials. Felt and leather are examples of surfaces which are basically developable but allow, compared to its size, for a little stretching or shrinking of the surface. In Figure 8 we approximate a 3D shape modeled from a piece of planar felt.

6.2.3 Surfaces of revolution as molds. Discrete geodesic parallel coordinates fulfilling the "constant ratio of velocities in $v$-direction"condition (Eqn. (5)), are surfaces isometric to rotational surfaces. For building such surfaces from flexible material, like metal sheets, one only has to build one mold, namely the corresponding surface of revolution. In fact, just a segment of that surface of revolution has to be built as mold, just large enough such that the largest panel fits on it. Note that one mold in the size of a segment (strip) of the surface of revolution (between two neighboring meridian curves) could exceed size limitations for molds. One has to produce as many molds as necessary to fill the segment of the rotational surface. Figure 16 illustrates which region of the surface corresponds to which part on the surface of revolution. For flat regions (vanishing Gaussian curvature) we do not have to build molds as the corresponding panels are simply developable (cf. Figure 17). For the radius of the surface of revolution we have some flexibility due to Proposition 4.3. The rendering in Figure 1 illustrates four different surface patches which are all isometric to the same surface of revolution. In the Frank Gehry-style building of Figure 1, the facades can be cladded with rectangular metal sheets which are actually not planar but isometric to some specific region of the mold surface of revolution. In Figure 19 we depict a short list of possible shapes that one can obtain as surfaces isometric to rotational surfaces.

6.2.4 Intrinsic repetitivity for strip models. Surfaces which are isometric to surfaces of revolution (Fig. 10, top-left) can be generated as a strip model by joining together isometric strips (Figure 18). This is an obvious advantage for the fabrication of such a doubly-curved surface as one must only manufacture several copies of a strip of the same shape from bendable material. We model these surfaces by discrete geodesic parallel coordinates (Figure 18 middle-row-left) with the additional constraint of an equal distribution of the strip width along all geodesic strips (7). Notice that our discrete geodesic strips do not incorporate any developability condition. However, for manufacturing purposes we have to unfold the strips into the 

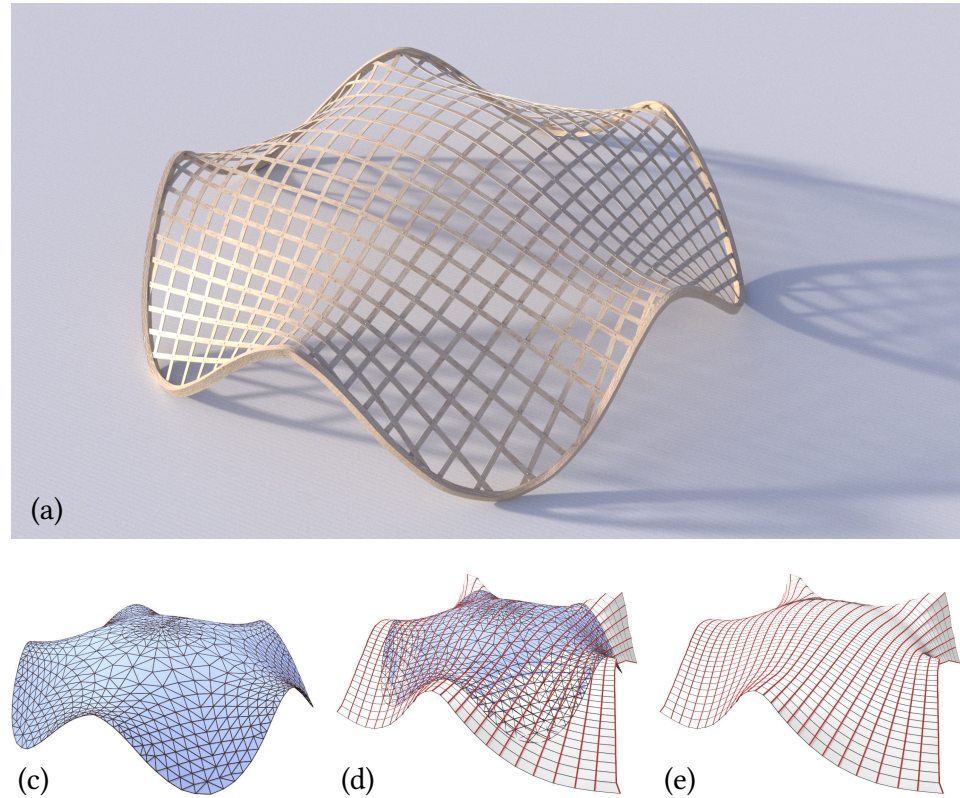

(e)

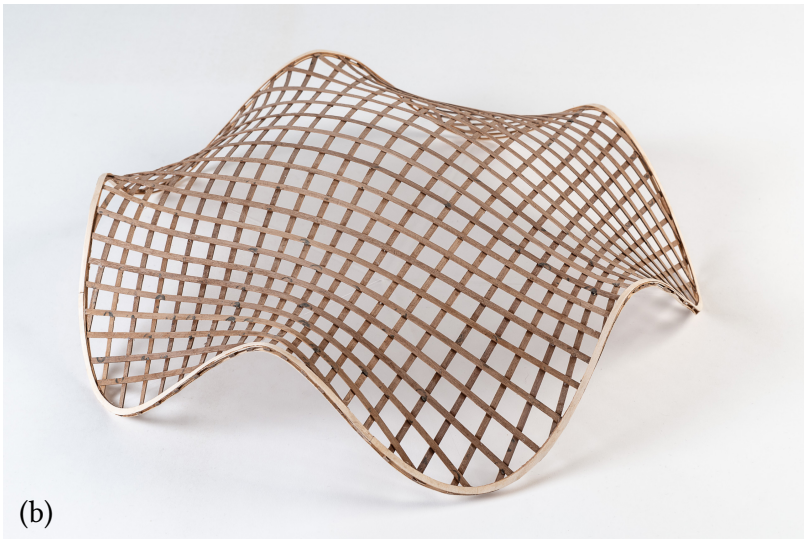

Fig. 14. Gridshell. We approximate a reference surface given as triangle mesh (c) with two different nets of discrete geodesic parallel coordinates, with the geodesics in two transversal directions and going beyond the reference surface. An overlay of the reference mesh with one of the two discrete geodesic parallel coordinates illustrates the approximation quality (d). One resulting net with discrete geodesic parallel coordinates is illustrated by (e). For the gridshell only the geodesics are built by thin elongated quadrilateral timber strips (a rendering (a) and a photo (b) of a real built timber strip model). The Hausdorff distance between the given mesh (bounding box diagonal length 25) and our geodesic parallel coordinate net is 0.031 .

plane. For that, we first transform each strip into a discrete developable strip (strip with planar quadrilateral faces). See Figure 18 (middle-row-right). We do that by first estimating the ruling directions by intersecting neighboring tangent planes and thus by computing the cross product $N \times N_{1}$, as also done in Figure 12 for

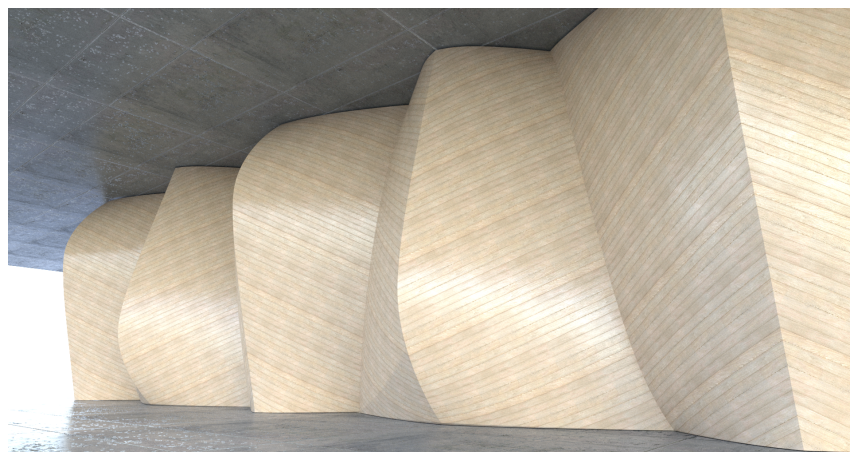

Fig. 15. Rendering of an interior wooden wall cladding. The shape obtained by bending planar wooden boards can be modeled by nearly developable surfaces since the material could slightly stretch or shrink while being bent.

Fig. 16. Surface of revolution as mold. Each quad panel on the deformed surface (right) is isometric to the quad on the rotational surface (left) with the same color. This way we obtain the doubly-curved panels in the style of the four surfaces from the Frank Gehry-type design in Fig. 1. the computation of the ruling vector field. In regions with almost parallel normal vectors (flat parts of the strip) we could theoretically choose any direction. So we average over the closest neighboring directions from non-flat parts of the strip. Then we intersect the two discrete geodesics of the strip with the plane spanned by the ruling vector and the normal vector and passing through the midpoint of $v$-parallel edges. In this way we generate almost planar quads along each strip. The development of the strips is done by unfolding

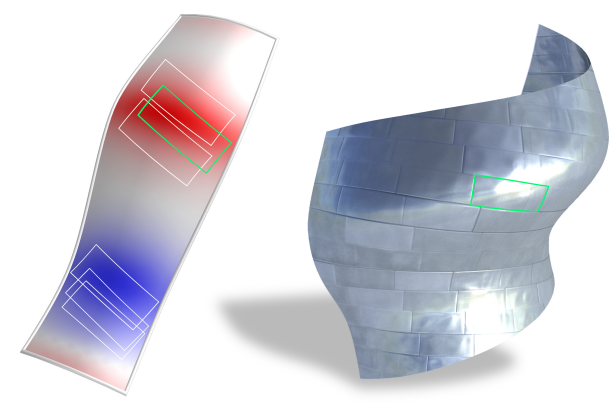

Fig. 17. Paneling of surfaces which are isometric to rotational surfaces (right). Each panel corresponds to a patch on the corresponding surface of revolution (left). With a green boundary we highlight such a pair. The segment of the surface of revolution has to be just big enough to be able to carry the largest panel. In Figure 16 we illustrate which regions on the surface correspond to which part (mold) on the rotational surface. In practice one would manufacture several molds to cover the area between two neighboring meridian curves due to size limitations for molds. In particular we don't need molds for regions with vanishing Gaussian curvature as these panels are flat. On the strip (left) we color surface regions with positive Gaussian curvature in red, negative in blue and close to zero in white. 


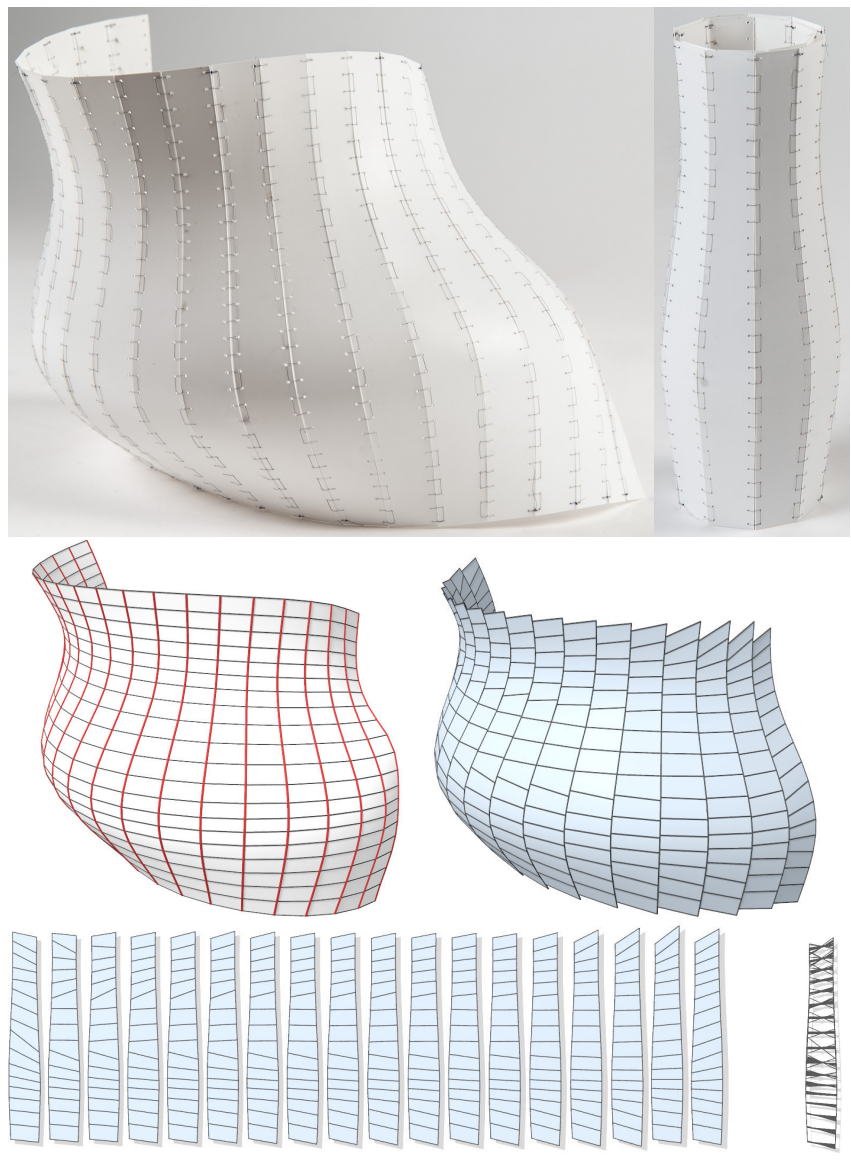

Fig. 18. Strip model of a surface isometric to a surface of revolution. Toprow: Photos of two deformations of a strip model of a surface of revolution fabricated by attaching (sewing up) congruent bendable strips (polystyrene). Middle-row-left: Model of the same surface by discrete geodesic parallel coordinates. Middle-row-right: To convert the geodesic strips to discrete developable strips we approximate the strips with piece wise planar faces. Notice that the ruling directions (edges transversal to the geodesics) are in general no longer orthogonal to the geodesics. Bottom-left: Development of each individual strip. Bottom-right: Overlay of all strips (without faces). Observe, how nicely the geodesics (vertical) boundaries match up, implying congruent strips.

the (almost) planar quads into the plane (Figure 18 bottom). An overlay of all developed strips shows nicely that the strips are almost congruent (Figure 18 bottom-right). The strong variation in the horizontal edges is due to the fact that rulings in developable surface can vary strongly while being bent isometrically.

6.2.5 Analysis. Due to the non-linearity of our optimization problem the algorithm converges to a local minimum which depends on the initialization. Consequently, there might appear self-intersections of the surface which are usually undesirable artefacts for applications and can be removed in our handle based editing mode. The influence of the chosen initialization mesh is bigger for our approximation tasks where we do not manually modify the mesh with handles while it is optimized for approximating a given surface.

The quadratic constraints for our variable vector $x \in \mathbb{R}^{3|V|+8|E|}$ can be written as system of quadratic equations $\varphi_{i}(x)=x^{\top} h_{i} x+$ $b_{i} x+c_{i}$ with $h_{i} \in \mathbb{R}^{(3|V|+8|E|) \times(3|V|+8|E|)}, b_{i} \in \mathbb{R}^{3|V|+8|E|}$, and $c_{i} \in \mathbb{R}$. In the following table we list the extent to which the solution fails to meet the constraints. We measure it by $R=\left(\sum_{i} \varphi_{i}(x)^{2}\right)^{1 / 2}$ after rescaling the mesh to an average edge length of 1 .

\begin{tabular}{|l|l|l|}
\hline Fig. no. & method & error $R$ \\
\hline \hline 1 & interactive & $2.75 \mathrm{e}-7$ \\
\hline 2 & interactive & $3.45 \mathrm{e}-7$ \\
\hline 8 & approximation & 0.29 \\
\hline $10-1$ & interactive & $2.10 \mathrm{e}-7$ \\
\hline $10-2$ & interactive & $1.32 \mathrm{e}-8$ \\
\hline$? ?$ & approximation & 0.031 \\
\hline
\end{tabular}

\subsection{Limitations and future research}

Limitations. Although the meshes that we work with (approx. 300 to 5000 vertices) quickly move towards their target shape already within the first iteration of the algorithm, our current implementation is not yet interactive. As our method has a main focus on material-aware fabrication, it would be nice to incorporate more properties of material behavior into the optimization. Apart from building a real gridshell (Figure ??) and a strip model of a surface isometric to a surface of revolution (Figure 18) we did not physically verify our methods, e.g., by running verification cycles with (other) physical simulations.

Future research. Material-aware geometric modeling is an area which offers a lot of room for future research. We have addressed some cases of working with materials that deform in an isometric way, but our handling of approximate isometries could be extended to precisely respect specific material properties such as limits in the bending and stretching behavior. We showed that intrinsic continuous symmetries have advantages in architectural applications. From a practical perspective, one could add here some tolerances and would no longer be so strictly confined to the class of surfaces which are isometric to surfaces of revolution. It is remarkable that these surfaces with a continuous intrinsic symmetry are related to surfaces which exhibit a form of extrinsic repetition. Julius Weingarten [1861] characterized surfaces which are isometric to rotational surfaces as focal surfaces of those surfaces which possess a relation between their principal curvatures. The latter so-called Weingarten surfaces are also interesting for applications, since they possess just a one-parameter family of curvature elements, which should lead to a substantial reduction of molds needed for the fabrication of panels. Optimizing an initial design surface towards more repetition of local shapes (curvature elements, locally approximating simple surface patches) is another interesting direction for future work.

Conclusion. We presented a new model for discrete geodesic parallel coordinates and its multifaceted potential for fabrication-aware design of architectural applications. We discussed how to model surfaces with intrinsically repetitive properties like cladding surfaces with equal strips and paneling surfaces with freeform appeal by doubly-curved panels for which only very few molds are necessary. 

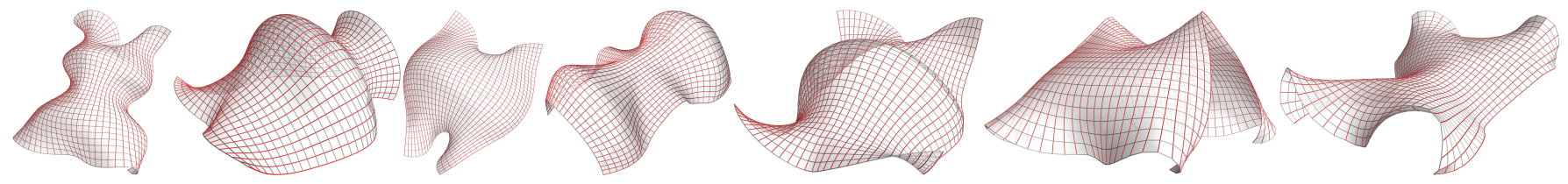

Fig. 19. The variety of surface patches isometric to surfaces of revolution depicted in this figure gives a hint on the wealth of possible shapes.

We introduced a design method for gridshell structures that can be built with elongated quadrilateral timber strips following geodesic curves. Our formulation as an optimization problem with at most quadratic constraints results in a near-interactive editing tool.

\section{ACKNOWLEDGMENTS}

The authors gratefully acknowledge the support of the Austrian science fund FWF under grants P 29981, I 2978, the WWTF under grant ICT15-082, the Chinese Scholarship Council and the National Natural Science Foundation of China (No. 11671068).

\section{REFERENCES}

Mirela Ben-Chen, Adrian Butscher, Justin Solomon, and Leonidas J. Guibas. 2010. On Discrete Killing Vector Fields and Patterns on Surfaces. Comput. Graph. Forum 29, 5 (2010), 1701-1711.

Fabio Bianconi and Marco Filippucci. 2019. Digital wood design. Lecture notes in Civil Engineering, Vol. 24. Springer.

Sebastien Callens and Amir Zadpoor. 2018. From flat sheets to curved geometries: Origami and kirigami approaches. Materials Today 21, 3 (2018), 241-264.

Wolfgang Carl. 2017. On semidiscrete constant mean curvature surfaces and their associated families. Monatsh. Math. 182, 3 (2017), 537-563.

Keenan Crane, Clarisse Weischedel, and Max Wardetzky. 2017. The Heat Method for Distance Computation. Commun. ACM 60, 11 (2017), 90-99.

Erik Demain and Joseph O'Rourke. 2007. Geometric Folding Algorithms. Cambridge University Press.

Levi H Dudte, Etienne Vouga, Tomohiro Tachi, and L Mahadevan. 2016. Programming curvature using origami tessellations. Nature materials 15, 5 (2016), 583.

Michael Eigensatz, Martin Kilian, Alexander Schiftner, Niloy Mitra, Helmut Pottmann, and Mark Pauly. 2010. Paneling Architectural Freeform Surfaces. ACM Trans. Graphics 29, 4 (2010), \#45,1-10.

Sebastian Finsterwalder. 1899. Mechanische Beziehungen bei der Flächendeformation. Jahresber. d. Deutschen Math.-Vereinigung 6 (1899), 43-90.

Konstantinos Gavriil, Alexander Schiftner, and Helmut Pottmann. 2018. Optimizing $\mathrm{B}$-spline surfaces for developability and paneling architectural freeform surfaces CoRR abs/1808.07560 (2018). arXiv:1808.07560 http://arxiv.org/abs/1808.07560

Elisa Lafuente Hernandez. 2015. Design and optimisation of elastic gridshells. Ph.D. Dissertation. Univ. of Arts, Berlin.

Joe Kahlert, Matt Olson, and Hao Zhang. 2011. Width-Bounded Geodesic Strips for Surface Tiling. The Visual Computer 27, 1 (2011), 45-56.

Mina Konaković, Keenan Crane, Bailin Deng, Sofien Bouaziz, Daniel Piker, and Mark Pauly. 2016. Beyond Developable: Computational Design and Fabrication with Auxetic Materials. ACM Trans. Graph. 35, 4, Article 89 (2016), 11 pages.

Mina Konaković, Julian Panetta, Keenan Crane, and Mark Pauly. 2018. Rapid Deployment of Curved Surfaces via Programmable Auxetics. ACM Trans. Graph. 37, 4, Article 106 (2018), 13 pages.

Wolfgang Kühnel. 2003. Differentialgeometrie (second ed.). Friedr. Vieweg \& Sohn, Braunschweig. viii+256 pages. Kurven-Flächen-Mannigfaltigkeiten.

Yang Liu, Helmut Pottmann, Johannes Wallner, Yong-Liang Yang, and Wenping Wang. 2006. Geometric modeling with conical meshes and developable surfaces. $A C M$ Trans. Graphics 25, 3 (2006), 681-689. Proc. SIGGRAPH.

Neil Meredith and James Kotronis. 2012. Self-detailing and self-documenting systems for wood fabrication: The Burj Khalifa. In Advances in Architectural Geometry 2012, L. Hesselgren et al. (Eds.). Springer, 185-198.

Ferdinand Minding. 1838. Ueber die Biegung krummer Flächen. 7. Reine Angew. Math 18 (1838), 365-368.

Jun Mitani and Hiromasa Suzuki. 2004. Making Papercraft Toys from Meshes Using Strip-based Approximate Unfolding. In ACM SIGGRAPH 2004 Papers (SIGGRAPH '04). ACM, New York, NY, USA, 259-263. https://doi.org/10.1145/1186562.1015711

Christian Müller and Johannes Wallner. 2013. Semi-discrete isothermic surfaces. Results Math. 63, 3-4 (2013), 1395-1407.
Maks Ovsjanikov, Jian Sun, and Leonidas J. Guibas. 2008. Global Intrinsic Symmetries of Shapes. Comput. Graph. Forum 27, 5 (2008), 1341-1348.

Jesus Perez, Miguel A. Otaduy, and Bernhard Thomaszewski. 2017. Computational Design and Automated Fabrication of Kirchhoff-Plateau Surfaces. ACM Trans. on Graphics 36, 4 (2017), 62.1-62.12. Proc. SIGGRAPH

Claudio Pirazzi and Yves Weinand. 2006. Geodesic lines on free-form surfaces: optimized grids for timber rib shells. In 9th World Conference on Timber Engineering. 72-79.

Konrad Polthier and Markus Schmies. 1998. Straightest Geodesics on Polyhedral Surfaces. Springer Berlin Heidelberg, Berlin, Heidelberg, 135-150.

Helmut Pottmann, Michael Eigensatz, Amir Vaxman, and Johannes Wallner. 2015. Architectural Geometry. Computers and Graphics 47 (2015), 145-164.

Helmut Pottmann, Oixing Huang, Bailin Deng, Alexander Schiftner, Martin Kilian, Leonidas Guibas, and Johannes Wallner. 2010. Geodesic Patterns. ACM Trans. Graphics 29, 4 (2010), \#43,1-10. Proc. SIGGRAPH.

Helmut Pottmann, Alexander Schiftner, Pengbo Bo, Heinz Schmiedhofer, Wenping Wang, Niccolo Baldassini, and Johannes Wallner. 2008. Freeform surfaces from single curved panels. ACM Trans. Graph. 27, 3 (2008), \#76,1-10. Proc. SIGGRAPH.

Michael Rabinovich, Tim Hoffmann, and Olga Sorkine-Hornung. 2018a. Discrete Geodesic Nets for Modeling Developable Surfaces. ACM Trans. Graph. 37, 2, Article 16 (2018), 17 pages.

Michael Rabinovich, Tim Hoffmann, and Olga Sorkine-Hornung. 2018b. The Shape Space of Discrete Orthogonal Geodesic Nets. ACM Trans. Graph. 37, 6, Article 228 (2018), 17 pages.

Dan Raviv, Alexander M. Bronstein, Michael M. Bronstein, and Ron Kimmel. 2010. Full and Partial Symmetries of Non-rigid Shapes. International fournal of Computer Vision 89, 1 (01 Aug 2010), 18-39.

Martin H Sadd. 2009. Elasticity: theory, applications, and numerics. Academic Press, Oxford.

Robert Sauer. 1970. Differenzengeometrie. Springer.

Eike Schling. 2018. Repetitive Structures - Design and construction of curved support structures with repetitive parameters. Ph.D. Dissertation. TU Munich.

Eike Schling, Martin Kilian, Hui Wang, Denis Schikore, and Helmut Pottmann. 2018 Design and construction of curved support structures with repetitive parameters. In Adv. in Architectural Geometry, L.H̃esselgren et al. (Ed.). Klein Publ. Ltd, 140-165.

Markus Schneider and Peter Mehrtens. 2012. Cladding freeform surfaces with curved metal panels - a complete digital production chain. In Advances in Architectural Geometry 2012, L. Hesselgren et al. (Eds.). Springer, 237-242.

J.A. Sethian. 1999. Level Set Methods and Fast Marching Methods: Evolving Interfaces in Computational Geometry, Fluid Mechanics, Computer Vision, and Materials Science. Cambridge University Press.

Dennis Shelden. 2002. Digital surface representation and the constructibility of Gehry's architecture. Ph.D. Dissertation. M.I.T.

Justin Solomon, Mirela Ben-Chen, Adrian Butscher, and Leonidas J. Guibas. 2011 Discovery of Intrinsic Primitives on Triangle Meshes. Comput. Graph. Forum 30, 2 (2011), 365-374.

Michael Spivak. 1979. A comprehensive introduction to differential geometry. Vol. II (second ed.). Publish or Perish, Inc., Wilmington, Del. $x v+423$ pages.

Oded Stein, Eitan Grinspun, and Keenan Crane. 2018. Developability of Triangle Meshes. ACM Trans. Graph. 37, 4, Article 77 (2018), 14 pages.

Chengcheng Tang, Pengbo Bo, Johannes Wallner, and Helmut Pottmann. 2016. Interactive Design of Developable Surfaces. ACM Trans. Graph. 35, 2, Article 12 (Jan. 2016), 12 pages.

Chengcheng Tang, Xiang Sun, Alexandra Gomes, Johannes Wallner, and Helmut Pottmann. 2014. Form-finding with Polyhedral Meshes Made Simple. ACM Trans. Graph. 33, 4, Article 70 (2014), 9 pages.

Julius Weingarten. 1861. Über eine Klasse aufeinander abwickelbarer Flächen. f. reine $u$. angewandte Mathematik 59 (1861), 382-393.

Walter Wunderlich. 1951. Zur Differenzengeometrie der Flächen konstanter negativer Krümmung. Österreich. Akad. Wiss. Math.-Nat. Kl. S.-B. IIa. 160 (1951), 39-77. 